\title{
Periarteriolar-Sparing Retinal Edema in Acute Central Retinal Artery Occlusion
}

\author{
Yoshifumi Ikeda Ichiya Sano Etsuko Fujihara Masaki Tanito \\ Division of Ophthalmology, Matsue Red Cross Hospital, Matsue, Japan
}

\section{Key Words}

Central retinal artery occlusion - Periarteriolar-sparing retinal edema - Prostaglandin E1 . Spectral-domain optical coherence tomography

\begin{abstract}
A 56-year-old man was referred to our hospital about $48 \mathrm{~h}$ after sudden onset of painless central visual loss in his right eye (OD) on a winter morning. He had a more than 25-year history of systemic hypertension and smoking. Funduscopic observation showed striated retinal whitening in the macular region, faint cotton-wool patches around the optic disc, and segmental narrowing of the retinal arteries near the optic disc edge. Based on the pattern of onset and fundus findings, he was diagnosed with vasospastic acute central retinal artery occlusion OD, and a venous drip injection of prostaglandin E1 was started immediately. Optical coherence tomography showed bands of high and low density in the inner retina arranged alternately in the edematous area; the bands corresponded to edematous and nonedematous areas, respectively, and geographically to venules and arterioles, respectively. The best-corrected visual acuity of 0.03 at referral improved to 0.5 , and the striated retinal edema and most cotton-wool patches resolved 1 month after onset. The oxygen pressure gradient in the capillary networks due to differences in the distance from the arterioles may be the mechanism of this unique periarteriolar-sparing retinal edema pattern.
\end{abstract}

\section{(C) 2015 The Author(s)}

Published by S. Karger AG, Basel

\section{Introduction}

Central retinal artery occlusion (CRAO) induced by emboli from carotid artery atherosclerosis, the most common cause of CRAO, is diagnosed based on sudden visual loss and

KARGER 125/s $\quad \begin{aligned} & \text { Yoshifumi Ikeda, MD, PhD } \\ & \text { Division of Ophthalmology } \\ & \text { Matsue Red Cross Hospital } \\ & 200 \text { Horo-machi, Matsue, Shimane 690-8506 (Japan) } \\ & \text { E-Mail yoshifumiikeda@hotmail.com }\end{aligned}$


Ikeda et al:: Periarteriolar-Sparing Retinal Edema in Acute Central Retinal Artery Occlusion

characteristic fundus findings including narrowing of the retinal arteries and macular whitening around the fovea that is typically seen as a cherry-red spot. Arterial spasms, a relatively rare cause of retinal artery occlusion, can cause branch retinal artery occlusion with complete superficial retinal opacification [1]. We report a case of CRAO that presumably was caused by a vasospastic mechanism and characterized by a unique striated pattern of retinal edema in the macula.

\section{Case Report}

A 56-year-old man was referred to our hospital about $48 \mathrm{~h}$ after sudden onset of painless central visual loss in his right eye (OD) on a winter morning. He had a more than 25-year history of systemic hypertension and smoking. At referral, the best-corrected visual acuity (BCVA) was $0.03 \mathrm{OD}$ and 1.2 in the left eye (OS), and the respective intraocular pressure values were 12 and $16 \mathrm{~mm} \mathrm{Hg}$. Funduscopic observation showed striated retinal whitening in the macular region, faint cotton-wool patches around the optic disc (fig. 1a), and segmental narrowing of the retinal arteries near the optic disc edge (fig. 1b). A horizontal foveal spectral-domain optical coherence tomography (SD-OCT) scan showed thickening of the inner retina (fig 1c). A horizontal SD-OCT scan of the edematous region 0.5 disc diameter above the fovea showed discrete distributions of inner retinal thickening with patchy increases in reflectivity in the inner retina (fig. 1d). A comparison of the color fundus (fig. 1a) and SD-OCT images (fig. 1d) showed that the hyperreflectivity coincided with the retinal opacification around the venules and the lower reflectivity coincided with the nonedematous retinal tissue around the arterioles. Based on the pattern of onset and fundus findings, we diagnosed incomplete acute CRAO OD and immediately started a venous drip injection of prostaglandin E1 (PGE1) [2]. Fluorescein angiography performed after the initial PGE1 drip showed an arm-to-retina circulatory time of $28 \mathrm{~s}$ and an early-phase filling defect in the small capillary networks in the macular region (fig. 1e). Doppler echocardiography did not show arterial narrowing or endothelial plaque in either carotid artery. According to a reported protocol [2], the patient was treated with intravenous PGE1 (80 $\mu \mathrm{g} /$ day) for 5 days and then oral PGE1 (30 $\mu \mathrm{g} /$ day) for 1 month. One month after onset, the BCVA improved to $0.5 \mathrm{OD}$ and the striated retinal edema and most cotton-wool patches resolved (fig. 1f). SDOCT showed marked thinning of the inner retina in the macula (fig. $1 \mathrm{~g}$ ) compared with the initial visit (fig. 1c).

\section{Discussion}

Typically, acute CRAO is characterized on fundus examination by a cherry-red spot surrounded by homogenous superficial retinal whitening, while cotton-wool patches are infrequently seen, and OCT shows thickening in the entire inner half of the sensory retina with blood supply from the central retinal arteries [3]. Thus, the appearance in the current case is atypical compared with the typical acute CRAO induced by a thrombus or embolus.

Based on the findings that included sudden visual loss, retinal edema, and a filling defect of the macular capillary networks accompanied by segmental constriction of the retinal arteries and cotton-wool patches, we diagnosed this case as an incomplete form of CRAO induced by arterial vasoconstriction. Cold stimulation [4] and smoking [5] can induce arterial vasoconstriction. Systemic hypertension can increase differences in retinal oxygen satura- 
Ikeda et al.: Periarteriolar-Sparing Retinal Edema in Acute Central Retinal Artery Occlusion

tion between the arterioles and venules [6]. Thus, winter onset, a history of systemic hypertension, and smoking might have been associated with the vasospastic CRAO in this case.

The fundus appearance in the current case seems identical to cases reported by Kurimoto et al. [7], who described three cases of incomplete CRAO resembling 'Purtscher-like retinopathy' with scattered macular edema and cotton-wool patches, although the authors did not fully discuss the mechanism of the development of the scattered macular edema. Using the currently available modalities, we found that the alternating arrangement of the inner retinal edema is associated geographically with the distribution of the retinal vessels, i.e., edema develops only around the retinal venules and no edema develops around the retinal arterioles. Accordingly, we propose that differences in oxygen saturation resulting in differences in the distances from the arterioles may explain the alternating pattern of retinal edema in incomplete CRAO. PGE1 administered systemically can improve the visual acuity if it is administered during the acute phase, i.e., typically within 1 day after the onset of acute CRAO $[2,8]$. The visual acuity in the current case recovered after administration of systemic PGE1 2 days after onset. Kurimoto et al. [7] reported a relatively good visual prognosis in their patients with incomplete CRAO. Thus, the effect of systemically administered PGE1 in the current case is inconclusive. To avoid recurrent CRAO, our patient continued to take oral PGE1 until 1 month after onset, although the need for sustained use of oral PGE1 is undetermined.

In conclusion, we observed a unique fundus appearance of striped retinal edema with faint cotton-wool patches in a patient with incomplete CRAO due to vasospasm. Differences in the oxygen pressure gradient in the capillary networks due to differences in the distances from the arterioles may be the mechanism of periarteriolar-sparing retinal edema.

\section{Statement of Ethics}

The patient provided written informed consent for publication of this case report and any accompanying images.

\section{Disclosure Statement}

The authors declare that there is no conflict of interest regarding the publication of this paper.

\section{References}

1 Abdul-Rahman AM, Gilhotra JS, Selva D: Dynamic focal retinal arteriolar vasospasm in migraine. Indian J Ophhalmol 2011;59:51-53.

2 Takai Y, Tanito M, Matsuoka Y, Hara K, Ohira A: Systemic prostaglandin E1 to treat acute central retinal artery occlusion. Invest Ophthalmol Vis Sci 2013;54:3065-3071.

-3 Ikeda F, Kishi S: Inner neural retina loss in central retinal artery occlusion. Jpn J Ophthalmol 2010;54:423429.

4 Nagaoka T, Mori F, Yoshida A: Retinal artery response to acute systemic blood pressure increase during cold pressor test in human. Invest Ophthalmol Vis Sci 2002;43:1941-1945.

-5 Morita S, Mizuno Y, Harada E, Nakagawa H, Morikawa Y, Saito Y, Katoh D, Kashiwagi Y, Yoshimura M, Murohara T, Yasue H: Differences and interactions between risk factors for coronary spasm and atherosclerosis - smoking, aging, inflammation, and blood pressure. Intern Med 2014;53:2663-2670.

6 Jani PD, Mwanza JC, Billow KB, Waters AM, Moyer S, Garg S: Normative values and predictors of retinal oxygen saturation. Retina 2014;34:394-401. 
Case Reports in

Ophthalmology

\begin{tabular}{l|l}
\hline Case Rep Ophthalmol 2015;6:390-393 \\
\hline DOI: 10.1159/000442175 & $\begin{array}{l}\text { (c) 2015 The Author(s). Published by S. Karger AG, Basel } \\
\text { www.karger.com/cop }\end{array}$ \\
\hline
\end{tabular}

Ikeda et al.: Periarteriolar-Sparing Retinal Edema in Acute Central Retinal Artery Occlusion

7 Kurimoto T, Okamoto N, Oku H, Kanbara Y, Etomi T, Tonari M, Ikeda T: Central retinal artery occlusion resembling Purtscher-like retinopathy. Clin Ophthalmol 2011;5:1083-1088.

8 Steigerwalt RD Jr, Cesarone MR, Pascarella A, De Angelis M, Nebbioso M, Belcaro G, Feragalli B: Ocular and optic nerve ischemia: recognition and treatment with intravenous prostaglandin E1. Panminerva Med 2011;53:119-124.
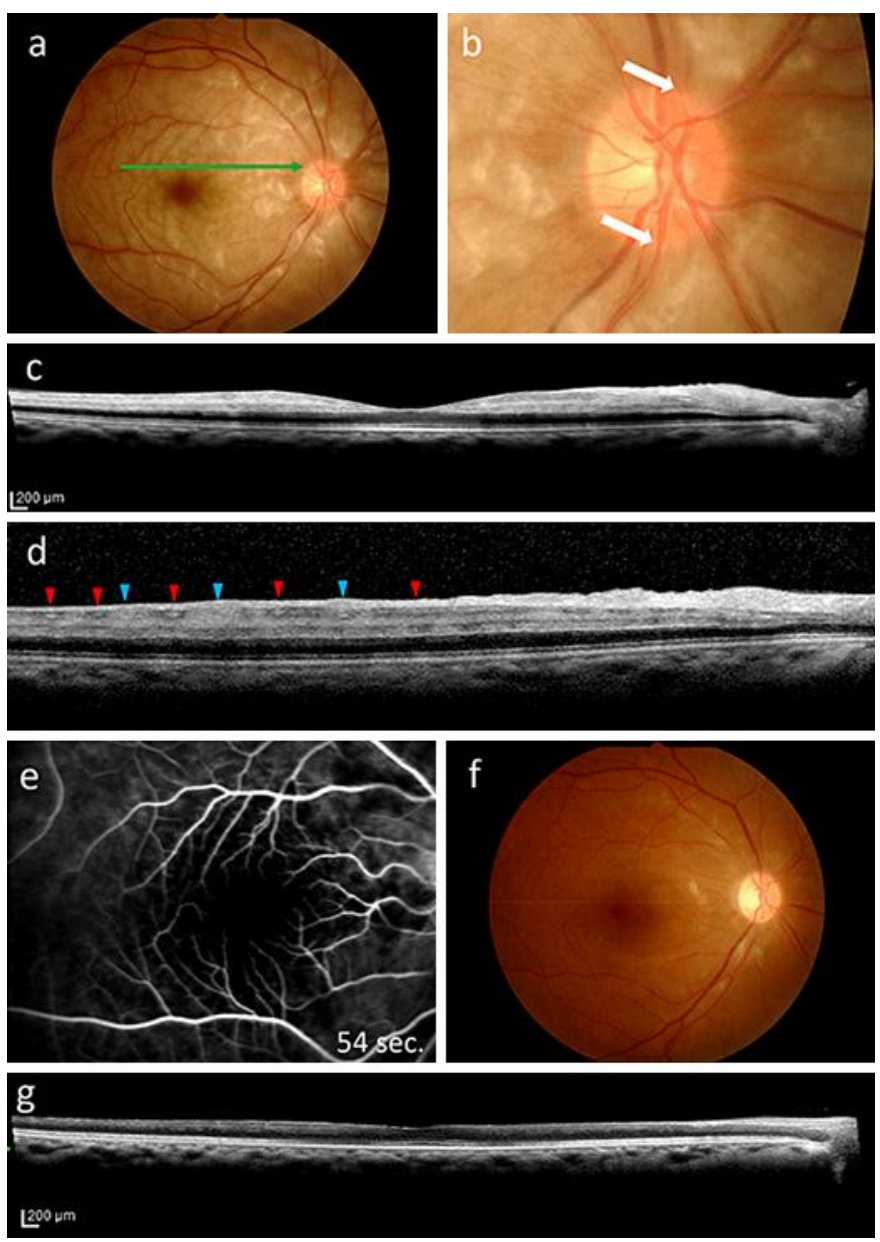

Fig. 1. Striated retinal whitening around the fovea and faint cotton-wool patches around the optic disc are observed at the initial visit (a). The green arrow corresponds to the scan direction in $\mathbf{d}$. Spastic arteries are seen near the optic disc (b, arrows). In a horizontal foveal SD-OCT scan, edematous changes that correspond to hyperreflectivity are seen in the inner retina (c). In a horizontal macular SD-OCT scan 0.5 disc diameter above the fovea, the hyperreflectivity and hyporeflectivity correspond to edematous and nonedematous areas of the inner retina, respectively (d). A comparison of $\mathbf{a}$ and $\mathbf{d}$ shows that the distributions of the hyperreflectivity and hyporeflectivity coincide with the distributions of the venules (blue arrowheads) and arterioles (red arrowheads), respectively. Fluorescein angiography performed at the initial visit shows a filling defect in the capillary networks in the macular region during the early phase (e). One month after the start of systemic PGE1 therapy, the striated retinal edema pattern resolved (f) and thinning of the inner retina is seen on the SD-OCT image $(\mathrm{g})$. 